\title{
BMJ Open Hypothermic oxygenated machine perfusion (HOPE) for orthotopic liver transplantation of human liver allografts from extended criteria donors (ECD) in donation after brain death (DBD): a prospective multicentre randomised controlled trial (HOPE ECD-DBD)
} Zoltan Czigany, ${ }^{1}$ Wenzel Schöning, ${ }^{1}$ Tom Florian Ulmer, ${ }^{1}$ Jan Bednarsch, ${ }^{1}$
lakovos Amygdalos, ${ }^{1}$ Thorsten Cramer, ${ }^{1}$ Xavier Rogiers, ${ }^{2}$ Irinel Popescu, ${ }^{3}$
Florin Botea, ${ }^{3}$ Jiří Froněk, ${ }^{4}$ Daniela Kroy, ${ }^{5}$ Alexander Koch, ${ }^{5}$ Frank Tacke,
Christian Trautwein, ${ }^{5}$ Rene H Tolba, ${ }^{6}$ Marc Hein, ${ }^{7}$ Ger H Koek, ${ }^{8}$
Cornelis H C Dejong, ${ }^{1,9}$ Ulf Peter Neumann, ${ }^{1,9}$ Georg Lurje ${ }^{1}$
To cite: Czigany Z, Schöning W, Ulmer TF, et al. Hypothermic oxygenated machine perfusion (HOPE) for orthotopic liver transplantation of human liver allografts from extended criteria donors (ECD) in donation after brain death (DBD): a prospective multicentre randomised controlled trial (HOPE ECD-DBD). BMJ Open 2017;7:e017558. doi:10.1136/ bmjopen-2017-017558

- Prepublication history for this paper is available online. To view these files please visit the journal online (http://dx.doi. org/10.1136/bmjopen-2017017558).

Received 1 May 2017

Revised 26 August 2017

Accepted 14 September 2017

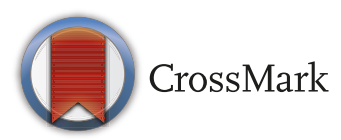

For numbered affiliations see end of article.

Correspondence to

Dr Georg Lurje;

glurje@ukaachen.de

\section{ABSTRACT}

Introduction Orthotopic liver transplantation (OLT) has emerged as the mainstay of treatment for end-stage liver disease. In an attempt to improve the availability of donor allografts and reduce waiting list mortality, graft acceptance criteria were extended increasingly over the decades. The use of extended criteria donor (ECD) allografts is associated with a higher incidence of primary graft non-function and/or delayed graft function. As such, several strategies have been developed aiming at reconditioning poor quality ECD liver allografts. Hypothermic oxygenated machine perfusion (HOPE) has been successfully tested in preclinical experiments and in few clinical series of donation after cardiac death OLT. Methods and analysis HOPE ECD-DBD is an investigatorinitiated, open-label, phase-II, prospective multicentre randomised controlled trial on the effects of HOPE on ECD allografts in donation after brain death (DBD) OLT. Human whole organ liver grafts will be submitted to 1-2 hours of HOPE $(n=23)$ via the portal vein before implantation and are going to be compared with a control group $(n=23)$ of patients transplanted after conventional cold storage. Primary (peak and $\Delta$ peak alanine aminotransferase within 7 days) and secondary (aspartate aminotransferase, bilirubin and international normalised ratio, postoperative complications, early allograft dysfunction, duration of hospital and intensive care unit stay, 1-year patient and graft survival) endpoints will be analysed within a 12-month follow-up. Extent of ischaemia-reperfusion (I/R) injury will be assessed using liver tissue, perfusate, bile and serum samples taken during the perioperative phase of OLT.
Strengths and limitations of this study

- HOPE ECD-DBD is a multicentre randomised controlled trial investigating the specific effects of Hypothermic oxygenated machine perfusion (HOPE) on extended criteria donor (ECD) organs in donation after brain death (DBD) transplantation.

- Tissue, blood, perfusate and bile-samples, retrieved for translational research, will enable an in-depth analysis of the effects of HOPE on ischaemiareperfusion injury and inflammation.

- The stratified randomisation model will allow us to achieve a homogeneous distribution of patients between treatment groups based on prognostic variables.

- Open-label design is considered a limitation of the study. Owing to the nature of the surgical procedure, it is not possible to blind the surgical team for the group allocation.

Ethics and dissemination The study was approved by the institutional review board of the RWTH Aachen University, Aachen, Germany (EK 049/17). The current paper represent the pre-results phase. First results are expected in 2018.

Trial registration number NCT03124641.

\section{INTRODUCTION}

Since Thomas Starzl's pioneering efforts in 1963, orthotopic liver transplantation 
(OLT) has evolved as the standard therapy for patients with end-stage liver disease. ${ }^{1}$ In 2015, approximately 1500 patients were listed for liver transplantation in Germany with only 894 transplants performed due to organ shortage. ${ }^{2}$ Several strategies for donor pool expansion are being pursued concurrently. These include the use of living donors, splitting of cadaveric livers for two recipients and the use of extended criteria donor (ECD) allografts for OLT. These ECD allografts, however, exhibit poor tolerance to ischaemia-reperfusion (I/R) injury, an important cause of liver damage. As such, $\mathrm{I} / \mathrm{R}$ injury is the underlying cause of graft dysfunction in ECD allografts and negatively affects the process of liver regeneration in surgical conditions including hepatic resections and OLT. $^{2}$

Accordingly, several strategies have been developed, aiming at reconditioning poor quality ECD allografts. ${ }^{3-7}$ Hypothermic oxygenated machine perfusion (HOPE) has been tested intensively in preclinical animal experiments. ${ }^{3}$ The positive effects of HOPE in this setting have been demonstrated among others to reduce the incidence of biliary complications, mitochondrial damage and level of cellular energy status. ${ }^{3}$ In the clinical setting, hypothermic oxygenated organ perfusion is performed in the transplant centre shortly before the actual implantation using an extracorporal organ perfusion system with full oxygen saturation over the portal vein. ${ }^{3}$ The first clinical study with HOPE was recently published by Dutkowski et al in a cohort of patients undergoing OLT with donation after cardiac death (DCD) allografts. ${ }^{8}$ In donation after brain death (DBD), the only legally accepted approach for organ donation in Germany and many other European countries, HOPE and its effect on early graft function and postoperative complications has not been reported yet. ${ }^{2}$

The purpose of this study is to test the effects of HOPE in a prospective multicentre randomised clinical trial (RCT) on ECD liver allografts in DBD liver transplantation compared with conventional cold storage (CCS; HOPE ECD-DBD). As such, we hypothesise that HOPE might have an organ reconditioning effect by substantially reducing early allograft injury in predamaged ECD organs. Primary (peak and $\Delta$ peak alanine aminotransferase (ALT) within 7 days) and secondary (eg, peak aspartate aminotransferase (AST), bilirubin (BR) and international normalised ratio (INR), lactate, postoperative complications, hospital stay, graft and patient survival) endpoints are going to be analysed. I/R injury and biomarkers of HOPE-induced subcellular responses will be assessed using liver tissue, perfusate, bile and serum samples during the perioperative phase of OLT. Follow-up is set at 12 months.

\section{METHODS AND ANALYSIS}

\section{Study type}

HOPE ECD-DBD is an investigator initiated, open-label, phase-II, prospective multicentre randomised controlled trial on ECD allografts in DBD OLT. Figure 1 summarises the trial design. The study protocol was written in accordance to the Consolidated Standards of Reporting Trials (CONSORT) and Standard Protocol Items: Recommendations for Interventional Trials (SPIRIT) recommendations.

\section{Eligibility}

Patients above 18 years of age, suffering from end stageliver disease and/or malignant liver tumours, listed for OLT and receiving ECD organs at the Department of Surgery and Transplantation, University Hospital RWTH Aachen, Aachen, Germany, and at other participating centres, are eligible for the study. Informed consent is obtained from all subjects participating in the trial by a qualified member of the study team in the outpatient clinic. In each centre, potential study participants on the OLT waiting list will be screened for defined inclusion and exclusion criteria and will be enrolled accordingly (figure 1). Allocation of liver allografts follows national (German Foundation for Organ Transplantation, The Belgian Transplantation Society, Romanian National Transplant Agency) and/or European (Eurotransplant (ET)) policies. The study will not influence the allocation procedure or cause any delay in the actual implantation procedure.

\section{Inclusion criteria}

Inclusion criteria are

1. signed informed consent

2. patients 18 years or older

3. patients suffering from end stage-liver disease and/or malignant liver tumours

4. listed for OLT

5. receiving ECD allografts.

\section{Exclusion criteria}

Exclusion criteria are

1. recipients of split or living donor OLT

2. previous OLT

3. combined transplantations (liver-kidney, liver-lung, etc.)

4. participation in other liver-related trials

5. the subject received an investigational drug within 30 days prior to inclusion

6 . the subject is unwilling or unable to follow the procedures outlined in the protocol

7. the subject is mentally or legally incapacitated

8. an inability to understand the procedures due to language barriers

9. family members of the investigators or employees of the participating department.

\section{Randomisation}

Randomisation is performed at arrival of the allograft at the transplant centre and after acceptance for transplantation, using an online randomising tool for clinical trials (www.randomizer.at), either by the principal investigator or, in case of absence, by a trained member of the study 


\section{Liver donation after brain death}

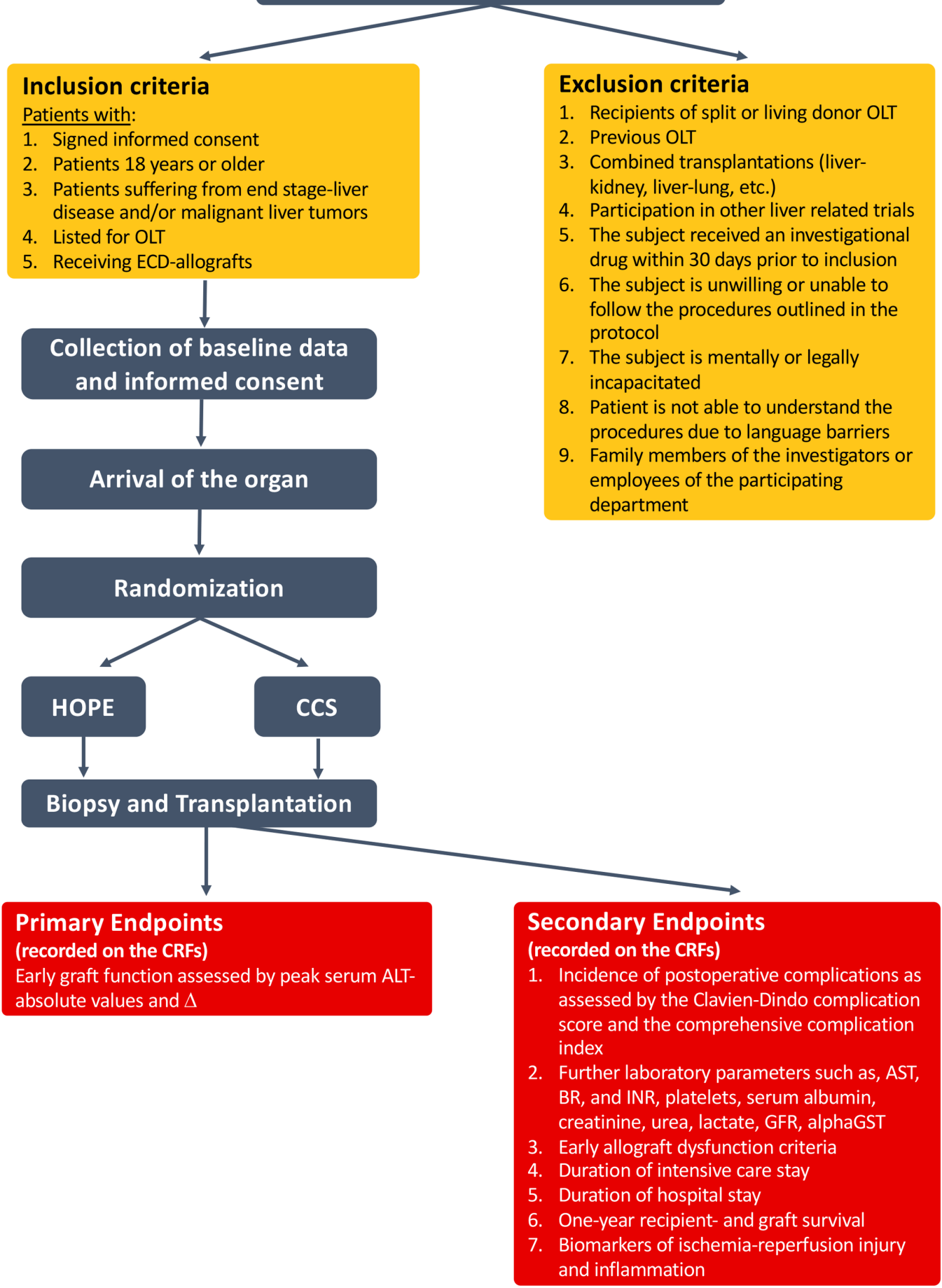

Figure 1 Study flowchart. alphaGST, alpha gluthation S-transferase; ALT, alanine aminotransferase; AST, aspartate aminotransferase; BR, bilirubin; CCS, conventional cold storage; CRF, case report file; ECD, extended criteria donor; GFR, glomerular filtration rate; HOPE, hypothermic oxygenated machine perfusion; INR, international normalised ratio; OLT, orthotopic liver transplantation.

team. ${ }^{9}{ }^{10}$ A stratified randomisation model will be used to ensure a balance of prognostic variables between the treatment groups. Expected cold ischaemia time and graft steatosis were selected for stratification. A median cold ischaemic time of 8 hours is based on our local OLT database (2010-2017) containing more than 320 OLTs.
Therefore, 8 hours of expected cold ischaemia time has been selected as a cut-off value for this study. For steatosis, we use $30 \%$ macrovesicular steatosis as a cut-off value for the stratification process as defined by the NASH Clinical Research Network Scoring System and validated as 
prognostic marker in liver transplantation by several authors. ${ }^{11-14}$

\section{Organ procurement, ECD criteria}

All ECD liver allografts will be retrieved by ET and/or national procurement teams. Following cross-clamping (in situ flushing of the abdominal organs and begin of cold ischaemia time), allografts will be removed and transported in a standardised fashion of CCS on packed ice $\left(4^{\circ} \mathrm{C}-6^{\circ} \mathrm{C}\right)$. Required data of the donor and the organ will be registered in a standard manner and will be transferred to a designated case report file (CRF).

Definition of ECD based on the recommendations of the German Medical Chamber (Bundesaerztekammer): ${ }^{15}$

1. donors 65 years of age and older

2 . intensive care therapy of the donor was required before donation for at least 7 days

3. obesity of the donor with a body mass index $>30 \mathrm{~kg} /$ $\mathrm{m}^{2}$

4. fatty liver (with histology) $>40 \%$

5. serum sodium $>165 \mathrm{mmol} / \mathrm{L}$

6. serum AST or ALT $>3$ times the upper limits of normal

7. serum $\mathrm{BR}>2 \mathrm{mg} / \mathrm{dL}$.

\section{HOPE versus CCS}

The present RCT comprises two groups, a perfusion (group 1; HOPE) and a control CCS (group 2) group.
Patients on the waiting list for OLT with proven written consent will be recruited.

In case of randomisation to group 1 , HOPE will be applied to the allograft in the operating room after regular organ procurement, transport and back-table preparation (figure 2) according to a protocol previously described by Dutkowski et al for DCD liver transplantation. ${ }^{8}$ HOPE (Liver Assist; Organ Assist b.v., Groningen, The Netherlands) will be applied through the portal vein for 1-(2) hour(s) (if recipient hepatectomy is prolonged, HOPE will be continued to avoid repeated static cold storage), with a perfusion rate of $0.1 \mathrm{~mL} / \mathrm{g}$ liver $/ \mathrm{min}$ in a pressured control system with $2-3 \mathrm{~mm} \mathrm{Hg}$. The $10^{\circ} \mathrm{C}$ perfusate $(3-4 \mathrm{~L})$ will be re-circulated (Belzer MPS, Bridge to Life, London, UK). Perfusate will be oxygenised with a $\mathrm{pO}_{2}$ of $60-80 \mathrm{kPa}$ by an oxygenator included as disposable in the setup. Perfusion parameters registered by the device will be stored automatically and evaluated for possible patterns. Immediately prior to perfusion, grafts are flushed with machine perfusion solution to wash out the residual HTK perfusate. Storage, management and use of the above-mentioned medical products will be carried out according to the manufacturer's guidelines. Patients in the CCS control group will undergo the clinically routine procedure with CCS using HTK solution (Custodiol, Dr Franz Köhler Chemie, Bensheim, Germany) according to institutional protocols.

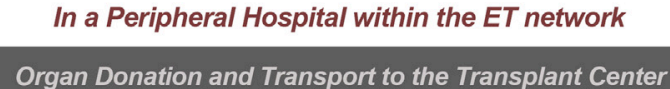

\section{In the Transplant Center \\ Back table HOPE vs. CCS}

HOPE, Liver Assist ® Device

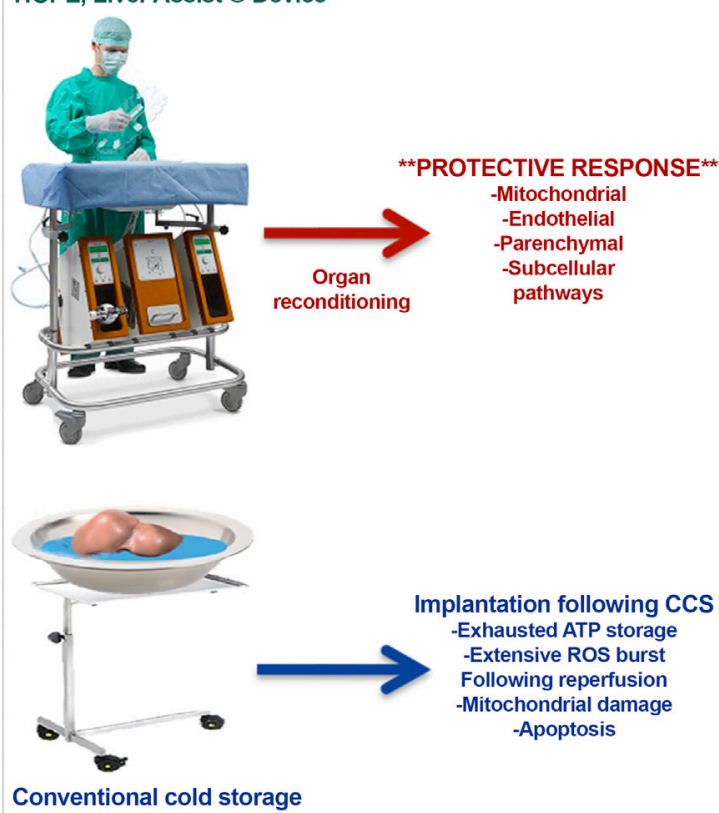

Figure 2 Hypothermic oxygenated machine perfusion (HOPE). First the donor organ is retrieved and transported to the transplant centre in a conventional way. In the transplant centre, the liver is connected to the liver assist device and HOPE is performed. Hypothetically, organ reconditioning with HOPE triggers multiple protective responses leading to decreased oxidative stress, improved energy reserves, reduced cell death. CCS, conventional cold storage; ET, Eurotransplant; ROS, reactive oxygen species. Adapted from Schlegel et al. ${ }^{24}$ 


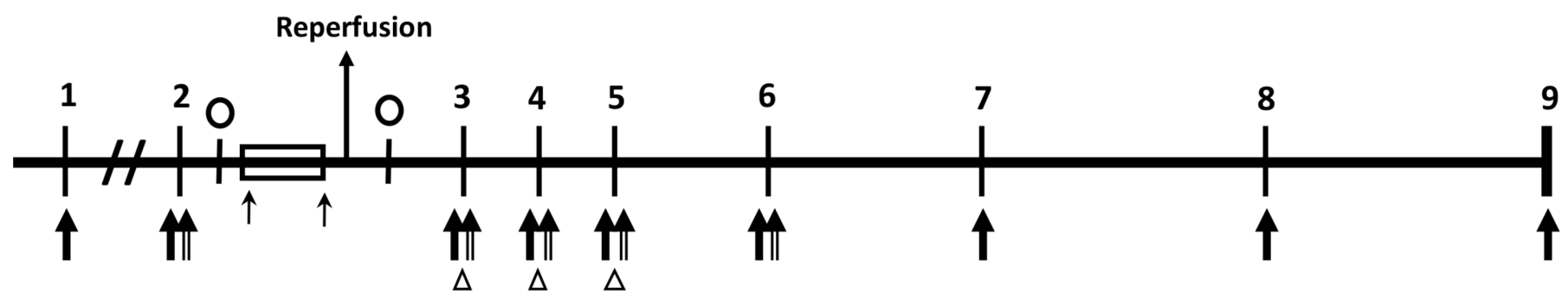

\section{Routine blood draw "If blood draw

Figure 3 Interventions and study visits. Arabic numbers represent the single study visits. Visit 1: screening, enrolment; visit 2: admission; visits 3, 4, 5: postoperative days 1, 2, 3; visit 6: seventh postoperative day; visit 7: discharge; visit 8: 6 months follow-up; visit 9: 12 months follow-up, final visit HOPE, hypothermic oxygenated machine perfusion.

\section{Orthotopic liver transplantation}

In centre 1, OLT will be performed using the total cava replacement technique with veno-venous bypass including the portomesenteric vascular bed using a roller pump system with an adjustable flow as previously described. ${ }^{1617}$ Biliary reconstruction will be performed as a side-to-side common bile duct anastomosis with T-tube insertion or primary Roux-en-Y hepaticojejunostomy in cases of primary sclerosing cholangitis as previously described. ${ }^{18}$ External centres will use their local standard techniques for OLT.

\section{Sample collection and storage}

I/R injury will be assessed using liver tissue samples taken on arrival of the organ (before HOPE or CCS) and at the end of implantation before closure of the abdomen, to evaluate the amount of I/R injury (figure 3 ). In total, two excision biopsies $\left(2 \mathrm{~cm}^{3}\right)$ will be harvested from the ECD liver allograft (segment III). Perfusate samples will be collected repeatedly during machine perfusion. Blood samples are taken as part of the daily routine during the perioperative and postoperative course of OLT (figure 3). Blood parameters of liver tissue damage and/or function (serum AST, ALT, BR, albumin, INR, alpha-gluthation S-transferase (GST), lactate) as well as kidney damage and/or function (creatinine, urea, glomerular filtration rate using the Chronic Kidney Disease Epidemiology Collaboration (CKD-EPI) equation) will be monitored. An additional $20 \mathrm{~mL}$ blood will be drawn on hospital admission and on postoperative days 1, 2, 3 and 7 (POD 1, POD 2, POD 3 and POD 7) and will be used for translational research (figure 3). Bile samples can be easily collected from the T-Drain during the first three PODs (only applicable for centre 1). All liver tissue, serum, perfusate, and bile samples will be directly snap-frozen in liquid nitrogen $\left(-80^{\circ} \mathrm{C}\right)$ and stored for 12 months after completion of the trial.

\section{Postoperative care and immunosuppression}

All patients are treated in accordance to our institution's routine clinical algorithm for OLT recipients. Apart for the ex vivo allograft perfusion (group 1, HOPE), patients will be treated according to standard operating procedures for perioperative medical, interventional and surgical OLT management. Patients after OLT will be monitored at the intensive care unit (ICU) during the early postoperative phase and later, depending on their individual recovery, transferred to regular ward.

The used immunosuppressive regimen is based on induction therapy with intravenous basiliximab and methylprednisolone followed by corresponding oral doses of prednisolone, tacrolimus and mycophenolate mofetil. ${ }^{16} 17$

\section{Study endpoints}

Primary endpoints

Early graft injury in DBD-OLT using ECD allografts undergoing HOPE or CCS, as assessed by peak ALT during the first 7 days post-OLT and by $\Delta$ peak ALT*. ${ }^{19}$

\section{Secondary endpoints}

1. Incidence of postoperative complications as assessed by the Clavien-Dindo complication score and the comprehensive complication index. ${ }^{20}$

2. Further laboratory parameters such as serum AST, BR, INR, platelet count, albumin, creatinine, urea, lactate, glomerular filtration rate using the CKD-EPI equation, alpha-GST.

3. Early allograft dysfunction as defined by the Olthoff criteria $(\mathrm{BR} \geq 10 \mathrm{mg} / \mathrm{dL}$ on day $7, \mathrm{INR} \geq 1.6$ on day 7 and ALT or AST>2000 IU / $\mathrm{L}$ within the first 7 days). ${ }^{21}$

4. Duration of intensive care stay.

5. Duration of hospital stay.

6. One-year recipient and graft survival.

7. Analysis of serum, tissue, bile and perfusate biomarkers as translational aspects of the project.

* $\triangle$ Peak ALT: To correct for an assumed washout effect of machine perfusion, besides the absolute values, relative changes of serum peak ALT will be assessed. ${ }^{19}$ Peak ALT will be corrected to the values measured in the routine blood analysis after reperfusion at the time point of admission to the ICU. 


\section{Power of the study}

A sample size of 23 patients per group $(\Sigma n=46)$ was calculated with the G*Power software (Heinrich-Heine-University, Düsseldorf, Germany) using the following settings: $\alpha=0.05 ; 1-\beta$ (power) $=0.8$; two-sided t-test, including $15 \%$ drop-out and invalid data; peak AST and ALT levels 48 hours post-transplantation. Power calculation was performed based on the previous data from Guarrera $e t$ $a l .{ }^{22} \mathrm{~A}$ reduction of $65 \%$ (AST) $-59 \%$ (ALT) is expected in the mean peak transaminase levels following machine perfusion treatment (peak AST: MP, 1154 $\pm 355.5 \mathrm{SD}$ IU/ mL, CCS, 3339 $\pm 3376.90 .1 \mathrm{SD}$ IU/mL; peak ALT: MP, $560.0 \pm 355.5 \mathrm{SD} \mathrm{IU} / \mathrm{mL}$, CCS, $1358 \pm 1208.4 \mathrm{SD} \mathrm{IU} / \mathrm{mL}){ }^{22}$

\section{Data collection and statistics}

All collected data are documented on CRFs and considered as source data. Members of the study team document the required information into the CRF system following previous training. A study database will be created based on the CRFs and data correction, record keeping, archiving and subsequently the destruction of study documents will be performed according to the international conference on harmonisation-good clinical practice (ICH-GCP) guidelines. Subjects will be informed about data protection and that data will be pseudonymised. Furthermore, data will be handed out to third party only anonymised. Encoded data will only be provided to authorised persons (clinical monitor, authorised study staff, authorities, institutional review board). The study will be prematurely terminated for an individual subject in case of study-related complications or if the subject withdraws informed consent. Values of $\mathrm{p}$ value less than 0.05 will be considered significant. Unpaired t-test and Mann-Whitney $\mathrm{U}$ test are going to be applied in case of normally and non-normally distributed data, respectively. For time course analysis of laboratory parameters, two-way analysis of variance is applied. Postoperative complications are assessed by the Fischer's exact test. For comparisons between Kaplan-Meier curves of 1-year graft and patient survival, the log-rank test is used.

Analysis of primary endpoints will be performed by an independent committee in a blinded fashion (Institute for Medical Statistics, RWTH Aachen).

\section{Safety considerations}

In this study, solely certified medical products (CE certification) will be used. Blood draws and liver biopsies during the transplant procedure are performed according to the clinical routine. Thus, no relevant study-related risks and no additional burden for the subjects are expected. Independent monitoring of data will be performed by the Clinical Trial Center Aachen (RWTH Aachen, Aachen, Germany). An interim analysis will be performed as soon as 12 patients are enrolled in each randomised group. The trial will be terminated immediately if one of the following criteria is fulfilled: significantly higher serum ALT levels $(p<0.001$ using Student's t-test) in the HOPE group compared with the CCS group (efficacy).
The proportion of grade $\geq$ III complications is significantly higher $(\mathrm{p}<0.05$, Fischer's exact test) in the HOPE group when compared with the CCS group (safety).

\section{Ethics}

This study will be conducted in compliance with the protocol, the currentversion of the Declaration of Helsinki, and good clinical practice guidelines (ICH-GCP) as well as all national legal and regulatory requirements. The institutional review board of the University RWTH Aachen has approved the study protocol, including consent form and patient information (EK 049/17). Members of the local study team have completed a course in good clinical practice as certified by the German Medical Chamber. The trial was registered on clinicaltrial.gov on 20.03.2017 (NCT03124641).

\section{Study group}

The study group of the HOPE ECD-DBD trial comprises the trial sponsor (GL, UPN, ZC) and the PI (GL) of the University Hospital RWTH Aachen (centre 1), the local investigators in Ghent (centre 2; XR), Bucharest (centre 3 ; IP, FB) and Prague (centre 4; JF). Each local investigator is in charge of the execution of the study and collection of data. The trial sponsor is responsible for randomisation, trial database, storage, statistical analysis and scientific writing. The trial will expectedly include the first patients (applicable for centre 1) in Q3 of 2017. External centres are expected to start the recruitment phase until the end of 2017.

\section{I/R injury and inflammation}

I/R injury, depleted energy reserves and oxidative stress play an important role in early graft dysfunction following liver transplantation of ECD liver grafts. ${ }^{34823-26}$ The translational research aim of this study is to determine the effects of HOPE on I/R injury, inflammation and energy household on human liver ECD allografts. Blood and liver tissue samples will be used to measure various parameters of inflammation (interleukins, tumour necrosis factoralpha, macrophage migration inhibitory factor), ${ }^{27} 28$ hepatocyte cell death (eg, circulating cytokeratin 18 fragments like M30 or M65), ${ }^{29}$ energy (ATP levels) ${ }^{30}$ and redox household (hemoxygenase-1, malondialdehyde). ${ }^{28}$ Luminometry, spectrophotometry, Luminex ${ }^{\circledR}$ assay, ELISA, Real-Time-PCR and western blot will be used for these analyses. Proteomics and metobolomics analysis will be performed on paired liver tissue samples to potentially identify early mediators of HOPE-mediated organ protection.

Biochemical parameters of biliary epithelial cell function and injury (BR, biliary $\mathrm{pH}$, bicarbonate, biliary glucose, lactate dehydrogenase, alkaline phosphatase, gamma-glutamyltransferase) will be assessed using standard laboratory methods. ${ }^{19} 31$

Due to the scarcity of clinical data regarding the utilisation of HOPE for quality assessment of liver grafts, it is still unclear whether this method is applicable for such 
quality predictions. Therefore, we will use the perfusion fluid to assess certain parameters which might have a predictive value in evaluating graft quality under hypothermic conditions $\left(\mathrm{pH}\right.$, lactate, $\mathrm{PO}_{2}, \mathrm{PCO}_{2}$, AST, ALT, $\mathrm{LDH}, \mathrm{GST}$ liver fatty acid binding protein levels). ${ }^{31-33}$

\section{DISCUSSION}

Liver transplantation is the treatment of choice for patients with end-stage liver disease. The need to obtain the optimal benefit from a limited number of organs that are available has prompted the expansion of allograft selection criteria (ECD allografts) aiming at increasing the donor pool and decrease overall waiting list mortality. ECD allografts exhibit poor tolerance to $I / R$ injury, a syndrome initiated on restoration of blood supply after cold and warm ischaemia yielding in endothelial and Kupffer cell activation, vasoconstriction, neutrophil infiltration and sinusoidal platelet aggregation. ${ }^{34} 35$

Technical innovations have been introduced to overcome these difficulties of ECD allograft usage in OLT. ${ }^{4}$ The implementation of machine perfusion for liver allografts was one of the most promising innovations in organ preservation over the last decade. ${ }^{4}$ In vivo and ex vivo machine perfusion of liver allografts has been tested intensively in preclinical studies, ${ }^{3}$ and the beneficial effects of HOPE have been demonstrated among others to reduce the incidence of biliary complications, the degree of mitochondrial damage and the level of cellular energy status. ${ }^{3}$ In a recent international-matched case analysis, Dutkowski et al demonstrated for the first time that HOPE treatment of DCD allografts significantly decreased graft injury compared with matched CCS livers regarding peak ALT levels (1239 vs $2065 \mathrm{U} / \mathrm{L}, \mathrm{p}=0.02$ ), intrahepatic cholangiopathy ( $0 \%$ vs $22 \%, \mathrm{p}=0.015)$ and overall biliary complications ( $20 \%$ vs $46 \%, \mathrm{p}=0.042)$, thus concluding that HOPE seems to offer beneficial effects in DCD allograft preservation. ${ }^{8}$ In DBD, the only legally accepted approach for organ donation in Germany and many other countries, HOPE and its effect on graft function and postoperative complications has not been reported yet. ${ }^{2}$ Currently, we identified four active clinical trials on clinicaltrials.gov using HOPE in liver transplantation. Two trials are non-randomised observational studies both with the enrolment of 10 patients for machine perfusion, using different perfusion systems (NCT03098043; NCT03031067). One trial (NCT02584283) is a multicentre RCT investigating the effects of portal and arterial perfusion HOPE (dual-HOPE) versus cold storage on biliary complications in DCD transplantation (table 1). Preliminary data with dual-HOPE have been reported recently in a case-control feasibility and safety study by the same group. ${ }^{36}$ The second RCT by Dutkowski et al (NCT01317342) is recruiting patients in DBD transplantation, however, also including non-ECD organs thus appearing less suitable to investigate the specific effects of HOPE in ECD allografts (table 1).

HOPE using single (portal vein only) or dual (portal vein and hepatic artery) perfusion is subject of ongoing debate. ${ }^{24}{ }^{36-40}$ Arguments for the use of dual perfusion are based predominantly on the better perfusion and preservation of the biliary system and reduced incidence of biliary complications in DCD transplantation. ${ }^{36}{ }^{39}$ However, according to some experimental data a complete end effective perfusion of the graft and the biliary system can also be achieved by using portal vein only perfusion. ${ }^{38}$ Due to the lack of any in vivo clinical evidence or convincing comparative preclinical data which would demonstrate the superiority of any of the two approaches, we decided to use the pragmatic approach and perfuse the liver grafts via the portal vein only. The favourable effects of portal vein only HOPE have already been successfully demonstrated in clinical liver transplantation by Dutkowski et al. ${ }^{8}$ Nevertheless, the comparison of single versus dual HOPE is of upmost clinical importance and should be addressed in future trials.

Although we have designed our trial carefully, non-blinding of the transplant team for the treatment

\begin{tabular}{|c|c|c|c|c|c|c|}
\hline Trial number & Study centre & Study type & Enrolment & Donor group & Primary endpoint & Comment \\
\hline $\begin{array}{l}\text { NCT03124641 } \\
\text { (present trial) }\end{array}$ & $\begin{array}{l}\text { RWTH Aachen } \\
\text { University, Aachen, } \\
\text { Germany }\end{array}$ & $\mathrm{RCT}$ & 46 & ECD-DBD & $\begin{array}{l}\text { Early graft function } \\
\text { (peak ALT level) }\end{array}$ & Recruiting \\
\hline NCT01317342 & $\begin{array}{l}\text { University of Zurich, } \\
\text { Zurich, Switzerland }\end{array}$ & $\mathrm{RCT}$ & $170^{\star}$ & $\begin{array}{l}\text { DBD } \\
\text { (ECD subgroup } \\
\text { analysis only) }\end{array}$ & $\begin{array}{l}\text { Major postoperative } \\
\text { complications (Clavien } \\
\text { grade } \geq \mathrm{III}) \text { and } \mathrm{CCl}^{*}\end{array}$ & Recruiting \\
\hline NCT02584283 & $\begin{array}{l}\text { University of } \\
\text { Groningen, } \\
\text { Groningen, } \\
\text { Netherlands }\end{array}$ & RCT & 156 & $\begin{array}{l}\text { DCD } \\
\text { (Maastricht } \\
\text { category III) }\end{array}$ & $\begin{array}{l}\text { Incidence of } \\
\text { symptomatic NAS }\end{array}$ & Recruiting \\
\hline
\end{tabular}

*Information based on personal communication.

ALT, alanine aminotransferase; CCI, comprehensive complication index; DBD, donation of the brain death; DCD, donation of the cardiac death; ECD, extended criteria donation; HOPE, hypothermic oxygenated machine perfusion; NAS, non-anastomotic biliary strictures; OLT, orthotopic liver transplantation; RCT, randomised controlled trial; RWTH, Rheinisch-Westfälische Technische Hochschule. 
groups is a limitation. This may be accounted to the nature of the surgical procedure and to pragmatic reasons, as HOPE and back-table preparation of the allograft are usually performed in the same operating room as the OLT procedure itself. The present trial has also some specific strengths. First, HOPE ECD-DBD focuses on patients solely receiving ECD allografts, a population we anticipate the best cost-benefit ratio from the utilisation of HOPE. Second, DBD is the most frequent source of ECD allografts in Europe. Third, we use a stratified randomisation model that allows us to achieve a homogeneous distribution based on prognostic variables of patients between the groups. Lastly, the results of the translational part of this study may deliver novel insights on the underlying subcellular effects of HOPE in human allografts.

\section{Author affiliations}

${ }^{1}$ Department of Surgery and Transplantation, University Hospital RWTH Aachen, Aachen, Germany

${ }^{2}$ Department of Solid Organ Transplantation, Ghent University Hospital and Medical School, Ghent, Belgium

${ }^{3}$ Department of General Surgery and Liver transplantation, Fundeni Clinical Institute, Bucharest, Romania

${ }^{4}$ Department of Transplantation Surgery, Institute for Clinical and Experimental Medicine, Prague, Czech Republic

${ }^{5}$ Department of Medicine III, University Hospital RWTH Aachen, Aachen, Germany

${ }^{6}$ Institute for Laboratory Animal Science and Experimental Surgery, University Hospital RWTH Aachen, Aachen, Germany

${ }^{7}$ Department of Anesthesiology, University Hospital RWTH Aachen, Aachen, Germany ${ }^{8}$ Section of Gastroenterology and Hepatology, Department of Internal Medicine, Maastricht University Medical Centre (MUMC), Maastricht, The Netherlands ${ }^{9}$ Department of Surgery, Maastricht University Medical Centre (MUMC), Maastricht, Netherlands

Contributors The initial study concept was derived from the initiating investigator study group (GL, ZC, UPN, RT, IA, JB). GL and ZC drafted the manuscript. ZC and GL performed the sample size estimation. WS, TFU, TC, XR, IP, FB, JF, DK, AK, FT, CT, $\mathrm{MH}, \mathrm{GHK}, \mathrm{CHCD}$ participated in designing the study, preparing the revised protocol and are investigators at the University Hospital RWTH Aachen or at the external centers. All authors were involved in revising the manuscript and approved the final version.

Funding This research project is supported by the START Program of the Faculty of Medicine, RWTH Aachen (691739, ID: 136/17).

Competing interests None declared.

Ethics approval Institutional review board of the RWTH Aachen, Aachen, Germany Approval number: EK 049/17.

Provenance and peer review Not commissioned; externally peer reviewed.

Open Access This is an Open Access article distributed in accordance with the Creative Commons Attribution Non Commercial (CC BY-NC 4.0) license, which permits others to distribute, remix, adapt, build upon this work non-commercially, and license their derivative works on different terms, provided the original work is properly cited and the use is non-commercial. See: http://creativecommons.org/ licenses/by-nc/4.0/

(c) Article author(s) (or their employer(s) unless otherwise stated in the text of the article) 2017. All rights reserved. No commercial use is permitted unless otherwise expressly granted.

\section{REFERENCES}

1. Starzl TE, Fung JJ. Themes of liver transplantation. Hepatology 2010;51:1869-84.

2. Tacke F, Kroy DC, Barreiros AP, et al. Liver transplantation in Germany. Liver Transpl 2016;22:1136-42.
3. Schlegel A, Kron P, Dutkowski P. Hypothermic machine perfusion in liver transplantation. Curr Opin Organ Transplant 2016;21:308-14.

4. Marecki H, Bozorgzadeh A, Porte RJ, et al. Liver ex situ machine perfusion preservation: A review of the methodology and results of large animal studies and clinical trials. Liver Transpl 2017;23:679-95.

5. Gurusamy KS, Gonzalez HD, Davidson BR. Current protective strategies in liver surgery. World J Gastroenterol 2010;16:6098-103.

6. Compagnon $\mathrm{P}$, Levesque $\mathrm{E}$, Hentati $\mathrm{H}$, et al. An oxygenated and transportable machine perfusion system fully rescues liver grafts exposed to lethal ischemic damage in a pig model of DCD liver transplantation. Transplantation 2017;101:e205-e213.

7. Ceresa CDL, Nasralla D, Knight S, et al. Cold storage or normothermic perfusion for liver transplantation: probable application and indications. Curr Opin Organ Transplant 2017;22:300-5.

8. Dutkowski P, Polak WG, Muiesan P, et al. First comparison of hypothermic oxygenated perfusion versus static cold storage of human donation after cardiac death liver transplants: an international-matched case analysis. Ann Surg 2015;262:764-71. discussion 770-1.

9. Lurje G, Raptis DA, Steinemann DC, et al. Cosmesis and body image in patients undergoing single-port versus conventional laparoscopic cholecystectomy: a multicenter double-blinded randomized controlled trial (SPOCC-trial). Ann Surg 2015;262:728-34. discussion 734-5.

10. Steinemann DC, Raptis DA, Lurje G, et al. Cosmesis and body image after single-port laparoscopic or conventional laparoscopic cholecystectomy: a multicenter double blinded randomised controlled trial (SPOCC-trial). BMC Surg 2011;11:24

11. Kleiner DE, Brunt EM, Van Natta M, et al. Design and validation of a histological scoring system for nonalcoholic fatty liver disease. Hepatology 2005;41:1313-21.

12. Westerkamp AC, de Boer MT, van den Berg AP, et al. Similar outcome after transplantation of moderate macrovesicular steatotic and nonsteatotic livers when the cold ischemia time is kept very short. Transpl Int 2015;28:319-29.

13. Chu MJ, Dare AJ, Phillips AR, et al. Donor hepatic steatosis and outcome after liver transplantation: a systematic review. $J$ Gastrointest Surg 2015;19:1713-24.

14. McCormack L, Dutkowski P, El-Badry AM, et al. Liver transplantation using fatty livers: always feasible? J Hepatol 2011;54:1055-62.

15. RichtlinienfürdieWartelistenführungundOrganvermittlungzurLebertransplantationgem. $\S 16$ Abs. 1 S. 1 Nrn. 2 u. 5 TPG. Bundesärztekamme:29.

16 Mossdorf A, Ulmer F, Junge K, et al. Bypass during liver transplantation: anachronism or revival? liver transplantation using a combined venovenous/portal venous bypass-experiences with 163 liver transplants in a newly established liver transplantation program. Gastroenterol Res Pract 2015;2015:1-7.

17. Kienlein S, Schoening W, Andert A, et al. Biliary complications in liver transplantation: Impact of anastomotic technique and ischemic time on short- and long-term outcome. World J Transplant 2015;5:300-9.

18. Neuhaus P, Blumhardt G, Bechstein WO, et al. Technique and results of biliary reconstruction using side-to-side choledochocholedochostomy in 300 orthotopic liver transplants. Ann Surg 1994;219:426-34.

19. Op den Dries S, Sutton ME, Karimian N, et al. Hypothermic oxygenated machine perfusion prevents arteriolonecrosis of the peribiliary plexus in pig livers donated after circulatory death. PLOS One 2014;9:e88521.

20. Slankamenac K, Graf R, Barkun J, et al. The comprehensive complication index: a novel continuous scale to measure surgical morbidity. Ann Surg 2013;258:1-7.

21. Olthoff KM, Kulik L, Samstein B, et al. Validation of a current definition of early allograft dysfunction in liver transplant recipients and analysis of risk factors. Liver Transpl 2010;16:943-9.

22. Guarrera JV, Henry SD, Samstein B, et al. Hypothermic machine preservation in human liver transplantation: the first clinical series. Am J Transplant 2010;10:372-81.

23. Dutkowski P, Schlegel A, de Oliveira M, et al. HOPE for human liver grafts obtained from donors after cardiac death. $J$ Hepatol 2014;60:765-72.

24. Schlegel A, Dutkowski P. Role of hypothermic machine perfusion in liver transplantation. Transpl Int 2015;28:677-89.

25. Schlegel A, Kron P, Dutkowski P. Hypothermic oxygenated liver perfusion: basic mechanisms and clinical application. Curr Transplant Rep 2015;2:52-62.

26. Schlegel A, Kron P, Graf R, et al. Hypothermic oxygenated perfusion (HOPE) downregulates the immune response in a rat model of liver transplantation. Ann Surg 2014;260:931-8. discussion 937-8.

27. Yang Z, Zhong Z, Li M, et al. Hypothermic machine perfusion increases A20 expression which protects renal cells against 
ischemia/reperfusion injury by suppressing inflammation, apoptosis and necroptosis. Int J Mol Med 2016;38:161-71.

28. Fu Z, Ye Q, Zhang Y, et al. Hypothermic machine perfusion reduced inflammatory reaction by downregulating the expression of matrix metalloproteinase 9 in a reperfusion model of donation after cardiac death. Artif Organs 2016;40:E102-E111.

29. Mazzolini G, Sowa JP, Canbay A. Cell death mechanisms in human chronic liver diseases: a far cry from clinical applicability. Clin Sci 2016;130:2121-38.

30. Westerkamp AC, Karimian N, Matton AP, et al. Oxygenated Hypothermic Machine Perfusion After Static Cold Storage Improves Hepatobiliary Function of Extended Criteria Donor Livers. Transplantation 2016;100:825-35.

31. Westerkamp AC, Mahboub P, Meyer SL, et al. End-ischemic machine perfusion reduces bile duct injury in donation after circulatory death rat donor livers independent of the machine perfusion temperature. Liver Transpl 2015;21:1300-11.

32. Jia JJ, Zhang J, Li JH, et al. Influence of perfusate on liver viability during hypothermic machine perfusion. World J Gastroenterol 2015;21:8848-57.

33. Monbaliu D, Liu Q, Libbrecht L, et al. Preserving the morphology and evaluating the quality of liver grafts by hypothermic machine perfusion: a proof-of-concept study using discarded human livers. Liver Transpl 2012;18:1495-507.
34. de Rougemont O, Dutkowski P, Clavien PA. Biological modulation of liver ischemia-reperfusion injury. Curr Opin Organ Transplant 2010;15:183-9.

35. Guan LY, Fu PY, Li PD, et al. Mechanisms of hepatic ischemiareperfusion injury and protective effects of nitric oxide. World $J$ Gastrointest Surg 2014;6:122-8.

36. van Rijn R, Karimian N, Matton APM, et al. Dual hypothermic oxygenated machine perfusion in liver transplants donated after circulatory death. Br J Surg 2017:104:907-17.

37. Schlegel A, Graf R, Clavien PA, et al. Hypothermic oxygenated perfusion (HOPE) protects from biliary injury in a rodent model of DCD liver transplantation. J Hepatol 2013;59:984-91.

38. Schlegel A, Kron P, De Oliveira ML, et al. Is single portal vein approach sufficient for hypothermic machine perfusion of DCD liver grafts? J Hepatol 2016;64:239-41.

39. Brüggenwirth IMA, Burlage LC, Porte RJ, et al. Is single portal vein perfusion the best approach for machine preservation of liver grafts? $J$ Hepatol 2016;64:1194-5.

40. Schlegel A, Kron P, de Oliveira ML, et al. Reply to 'Is single portal vein perfusion the best approach for machine preservation of liver grafts?'. J Hepatol 2016;64:1195-6. 